\title{
Decompressive effects of draining tube on suppurative and sclerosing osteomyelitis in the jaw
}

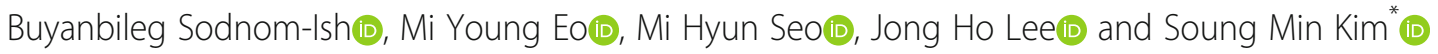

\begin{abstract}
Background: Osteomyelitis (OM) in the jaw is an inflammatory disease of osseous tissue that begins in the medullary space and progressively expands to the cortical portion of the bone, the Haversian system, the periosteum and the overlying soft tissue. Despite advances in dental and medical care, OM persists and is of important concern in modern medicine. Active negative pressure is known to prevent post-operative hematoma; decrease the number of bacterial pathogens, accumulation of toxins, and necrotic tissue; and promote osteogenesis and angiogenesis with the use of a draining tube such as the Jackson-Pratt (JP) or Hemovac. The purpose of this study was to assess the effectiveness of decompression for the treatment of OM in the jaw.
\end{abstract}

Methods: This retrospective study included a total of 130 patients, 55 patients with sclerosing OM and 75 patients with suppurative OM were included. The radiographic bone densities expressed as a grayscale values (GSVs), were measured using an easy digitalized panoramic analysis (EDPA) method, processed on the conditional inference tree, generated by the R program ${ }^{\oplus} 3.2 .3$ with a probability of $96.8 \%$. Rectangle annotation analysis of INFINITT PACS ${ }^{\circledR}$ (INFINITT Healthcare, Seoul, Korea) of $50 \mathrm{~mm}^{2}$ was determined as the region of interest (ROI). Student's t-test and ANOVA were used to determine significance $(p<0.05)$.

Results: Significant changes was observed between radiographic bone density in the sclerosing type with drain and without drain at the six-month and one-year follow-up $(p<0.05)$. Significant difference was demonstrated between the suppurative OM with drain and without drain groups at the one-year follow-up $(p<0.05)$.

Conclusion: The OM groups with drain exhibited more enhanced bone density compared to the groups without drain at the six-month and one-year follow-ups. The drain insertion for decompression is effective for the management of sclerosing and suppurative OM. It is recommended to implement it for the management of OM.

Keywords: Decompression, Suppurative osteomyelitis, Sclerosing osteomyelitis, Easy digitalized panoramic analysis (EDPA), Region of interest (ROI)

\footnotetext{
* Correspondence: smin5@snu.ac.kr; smin_kim@msn.com

Department of Oral and Maxillofacial Surgery, Dental Research Institute, School of Dentistry, Seoul National University, 101 Daehak-ro, Jongno-gu, Seoul 03080, South Korea
}

C C The Author(s). 2021 Open Access This article is licensed under a Creative Commons Attribution 4.0 International License, which permits use, sharing, adaptation, distribution and reproduction in any medium or format, as long as you give appropriate credit to the original author(s) and the source, provide a link to the Creative Commons licence, and indicate if changes were made. The images or other third party material in this article are included in the article's Creative Commons licence, unless indicated otherwise in a credit line to the material. If material is not included in the article's Creative Commons licence and your intended use is not permitted by statutory regulation or exceeds the permitted use, you will need to obtain permission directly from the copyright holder. To view a copy of this licence, visit http://creativecommons.org/licenses/by/4.0/ The Creative Commons Public Domain Dedication waiver (http://creativecommons.org/publicdomain/zero/1.0/) applies to the data made available in this article, unless otherwise stated in a credit line to the data. 


\section{Background}

Decompression and marsupialization are the major treatment options for odontogenic cysts. The efficacy of decompression has been widely demonstrated in odontogenic cystic conditions [1, 2]. Although the terms marsupialization and decompression have been used interchangeably, they are based on different concepts. Decompression effects can be classified as either passive (marsupialization and decompression) or active (negative pressure drainage) depending on the type of draining system used and the resulting induced negative pressure - low continuous, low intermittent, or high suction drainage [1]. Etymologically, the term marsupialization is derived from the Greek word marsupial meaning "pouch" [3]. Marsupialization is conversion of the cyst into a pouch, typically through suturing of the lining to the oral mucosa, so that the epithelial lining is left in situ [4]. Decompression on the other hand is the use of any draining tube (rubber tube, saliva ejector, catheter, anesthetic tube) to maintain patency between the inner contents of the cyst and the exterior environment [1, 5].

Decompression is based on the rationale that decreasing intraluminal pressure causes peripheral bone growth, changes the pathological environment, preserves anatomical structures, and prevents pathological fractures [6]. Recently, a two-way decompression device composed of two conduits, one of which is used for irrigation and the other for decompression has been introduced [2]. Castro-Nunez used an active negative pressure drainage apparatus connected to the internal compartment of the cyst to promote bone regeneration. The terms distraction sugosteogenesis and sugosteogenesis have been coined to define this negative pressureinduced osteogenesis [6].

Osteomyelitis (OM) in the jaw is an inflammatory disease of osseous tissue that begins in the medullary space and progressively expands to the cortical portion of the bone, the Haversian system, the periosteum and the overlying soft tissue. In the literature, the term OM has been used to describe multiple entities with different pathophysiological and clinical courses, resulting in confusion. The classification of OM varies among medical fields and is further obscured by radiographic, anatomic and etiological factors [7].

$\mathrm{OM}$ is typically caused by odontogenic infection or trauma. The odontogenic origin provides a direct pathway to the bone through pulpal infection or periodontal disease. This process begins with bacterial spread to the maxillary and mandibular bones, resulting in a bacteriainduced inflammatory disease [8]. Among the causative pathogens, Staphylococcus aureus, a facultative anaerobic gram-positive bacterium, is the most predominant in OM cases [9].
Among the various classification systems used for OM in the jaw, the Zurich classification is currently the most widely accepted. The Zurich Classification System differentiates the disease into three distinct entities: (1) acute OM, (2) secondary chronic OM, and (3) primary chronic (non-bacterial) OM [10]. The first two categories are characterized largely by suppurative nature, which is associated with sequestration and fistula formation [7]. Acute OM of the jaw develops over the course of several days to a few weeks and demonstrates a progressive onset of systemic symptoms, including fever, lymphadenopathy, leukocytosis, and edema formation in the affected region. Chronic OM is a relapsing and recurrent infection that progresses over months to years and is characterized by low-grade inflammation, presence of sequestration, new bone apposition and formation of fistulous tracts [11].

In the literature, two main variants of chronic OM are described. The suppurative variant has characteristics including presence of pus and or/fistulas and or/sequestrations that distinguish them from the non-suppurative variant, which is composed of chronic inflammatory processes of unknown etiology. The sclerosing variant is defined as an inflammatory bone condition of an uncertain origin even though it is generally believed that infections are of etiological significance. Radiographically, it is characterized by sequestra, sub-periosteal new bone formation, cortical defects, mixed sclerotic and osteolytic lesions are present and increased density of the medullary bone [12].

Despite advances in dental and medical care, OM persists and is of important concern in modern medicine. Consistent with the findings from previous authors, the prevalence of $\mathrm{OM}$ of the jaw seems to have increased recently $[11,13]$. In addition, systemic diseases like diabetes, which predisposes individuals to OM partly due to decrease in vascularity of the bone, are becoming more common [13]. Due to the wide variety of classification systems, multiple surgical options, and unlimited options of antibiotic therapy, even the most experienced surgeons can have difficult deciding on optimal treatment for $\mathrm{OM}$ of the jaw. Although antibiotic therapy has helped augment such treatment, surgical treatment remains the main therapeutic interventions and includes debridement, decortication, and resection [13].

Following surgical debridement of necrotic bone tissue, placement of an irrigation and drainage system is important. Despite aggressive surgical therapies, the infection may remain as a deep seated and wellestablished condition harboring necrotic tissue that exhibits resistance to antibiotics and the immune system. Vacuum-assisted closure (VAC) or negative pressure wound therapy is often used in nearly all surgical fields for complex wounds to eliminate pooled blood, serum, 
and dead space except during head and neck surgeries. VAC is seldom used in this field due to the complexity of the anatomical structures and conjunction of head and neck wounds with heavy bacterial burdens such as orocutaneous fistulae [7]. With installation of saline solutions, this treatment method is even more effective in infected soft-tissue wounds through enhancement of wound healing and reduction of wound infections $[8,9]$. Ceasar et al. reported use of a closed double-lumen suction irrigation system in chronic OM cases involving long bones. The suction and drainage aspects of this system allow for a "safety net" where the microbiology, volume, and appearance of the drainage fluid can be analyzed. Clear drainage indicates formation of healthy granulation tissue; if cloudy drainage persists, further surgical debridement must be undertaken, making way for proactive surgery. Other advantages included recovery of additional organisms from deep tissues [14]. A subjective method for evaluating the bone healing process in cystic lesions is associated with imprecise results and produces a significant bias that can be prevented by using the computerized analysis method [15]. Therefore, we implemented a picture archiving and communication system INFINITT PACS $^{\circ}$ (INFINITT Healthcare, Seoul, Korea) to measure radiographic bone density.

The purpose of this retrospective study was to quantitatively evaluate the effectiveness of decompression in treatment of chronic $\mathrm{OM}$ in the jaw using decompression with drain. We also discussed management updates about OM pathology and present management trends.

\section{Methods}

\section{Patients and study design}

From January 2009 through January 2020, 130 patients with OM who underwent treatment at Seoul National University Dental Hospital were retrospectively analyzed. $\mathrm{OM}$ of the jaw was classified as either suppurative or sclerosing type [16]. All panoramic radiographs were obtained with an Orthopantomograph OP100 (Instrumentarium Corp., Helsinki, Finland). The current study and its access to patient records were ethically approved by the Seoul National University Institutional Review Board (S-D20160039). All patients with $\mathrm{OM}$ in the jaw and odontogenic lesion were diagnosed by one oral and maxillofacial surgeon and included into the retrospective study with no age limit. Diagnoses for OM in the jaw were established based on patient medical history, clinical evaluation, radiological findings, and biopsy results.

Patients with OM were classified into four groups: sclerosing type with drain, sclerosing type without drain, suppurative type with drain, and suppurative type without drain.

\section{Inclusion criterion}

1) Patients with $\mathrm{OM}$ of the jaw who were treated at our institution between January 2009 and January 2020.

2) Patients diagnosed with sclerosing $O M$ and suppurative OM in the jaw.

3) Patients with full clinical data, including the periodic panoramic radiograph and laboratory data.

\section{Exclusion criteria}

1) Patients with insufficient medical records for analysis such as panoramic radiographs, laboratory data. and who were lost during the follow-up period.

2) Patients diagnosed with acute $\mathrm{OM}$ and other types of OM, such as the osteoradionecrosis, Bisphosphonate-related osteonecrosis of the jaw (BRONJ), Medication-related osteonecrosis of the jaw.

3) Patients who underwent partial resection and reconstruction.

\section{Treatment protocol and radiographic assessment}

Surgical therapy is one of the major pillars for treatment of acute and secondary chronic OM. Surgical debridement of infected tissue and removal of infectious foci were performed. Based on progression and pathology of the disease, we performed surgical therapy with minor surgical procedures such as removal of infected teeth, dental implants, fistulectomy, sequestrectomy, saucerization, and decortication. Following debridement of the infected tissue, we placed a silastic and penrose drain for passive drainage and a Hemovac or Jackson-Pratt drain for active negative pressure to drain post-operative exudate and promoted bone regeneration in the decompression groups of sclerosing type with drain and suppurative type with drain. The average amount of pressure measured in Jackson-Pratt drain was less than $100 \mathrm{mmHg}$, while the Hemovac drain generated $71 \pm 4$ $\mathrm{mmHg}$. The criterion for selecting the type of drain was based on the extent of the surgery and degree of marrow bone exposure. For extensive surgeries resulting in large amount of defect, a suction drain is more appropriate choice for the excess of removal of post-operative exudate. In smaller defects, a general tube is usually implemented.

When placing the decompressive drains in surgical wound area, the drains in situ should be placed between the soft tissue and the bone wall, avoiding from placing it into the bone cavity. This will serve as an easy method for fixture using suturing to the soft tissues and easy 
removal. Passive drainage and active negative pressure drainage were applied 1-5 days post-operatively.

\section{Bone density measurement method}

The radiographic bone densities expressed as grayscale values (GSV) were measured using an easy digitalized panoramic analysis (EDPA) method. Park et al. reported a method for early diagnosis and classification for $\mathrm{OM}$ in the jaw, which was processed with a decision making tree generated on $\mathrm{R}$ program ${ }^{\circ}$ version 3.2.3 for classifying the target group into subgroups based on the decision making tree with a probability of $96.8 \%$ [17]. We measured the GSV of the two groups on a fixed timeline. The measurements were collected immediately postoperatively and at three-month, six-month, and one-year follow-ups. We used INFINITT PACS ${ }^{\circ}$ (INFINITT Healthcare, Seoul, Korea) to measure bone density, where the average and standard deviation of the pixel values can be measured within the ellipse, rectangle, or arbitrary shape of choice. Rectangle annotation analysis was used as a standard length and width for each measurement. A standard rectangular lesion area of approximately $50 \mathrm{~mm}^{2}$ was defined as the region of interest (ROI), designated at the center of the OM lesion (Fig. 1a). Areas of teeth, titanium plates, dental implants, and any other foreign bodies were excluded. The bone density of the contralateral bone in the healthy region was measured as a reference value to determine the absolute value since there was no standardization of absolute value due to an error converting three-dimensional (3D) data into two-dimensional (2D) data, which depends on the posture and timing of the radiographic image. The difference between the ROI and the healthy contralateral site was calculated immediately postoperatively, 3 months later, 6 months later, and 1 year later to evaluate bone healing in the affected areas. In the panoramic radiograph, minimum, maximum, and average variables are presented in the histogram with values ranging from -240 to 2640 , where low radiographic density is expressed as radiopacity, and high radiographic density is expressed as radiolucency. For measurement, we used the dependent average variable of radiographic bone density [17].

\section{Laboratory analysis}

We retrospectively reviewed the medical records of all patients preoperatively and postoperatively. The hemoglobin $(\mathrm{Hb})$, hematocrit (Hct), white blood cells (WBC), absolute neutrophil count (ANC), and erythrocyte sedimentation rate (ESR) from the standard routine laboratory analysis were obtained 1-7 days before surgery. The normal reference levels of $\mathrm{Hb}$, Hct, WBC, ANC, and ESR were $12 \sim 16 \mathrm{~g} / \mathrm{dL}, 39-52 \%, 4 \sim 10 \times 10^{3}$ / $\mu \mathrm{l}, 1800 \sim 7000 / \mu \mathrm{l}$, and $0 \sim 20 \mathrm{~mm} / \mathrm{hr}$., respectively, at our institution. The postoperative laboratory results were compared with the preoperative results.

\section{Statistical considerations}

All data were collected using Excel (Microsoft, USA), and statistical analysis was performed using SPSS $25.0^{\circ}$ (SPSS Software Company, Chicago, IL, USA). Student's t-test and ANOVA were used to determine significance, defined as $0.05(p<0.05)$, and in the comparisons between mean values.

\section{Results}

\section{Patient and treatment data}

Based on the inclusion and exclusion criteria, a total of 43 patients were analyzed, in which the 23 patients were diagnosed with sclerosing $\mathrm{OM}$, while 20 patients were diagnosed with suppurative OM. Among them, 12 cases were males and 31 were female patients, with a mean age of $64.4 \pm 14.6$ ranging from 12 to 87 years. Regarding site, the right posterior mandible was the most

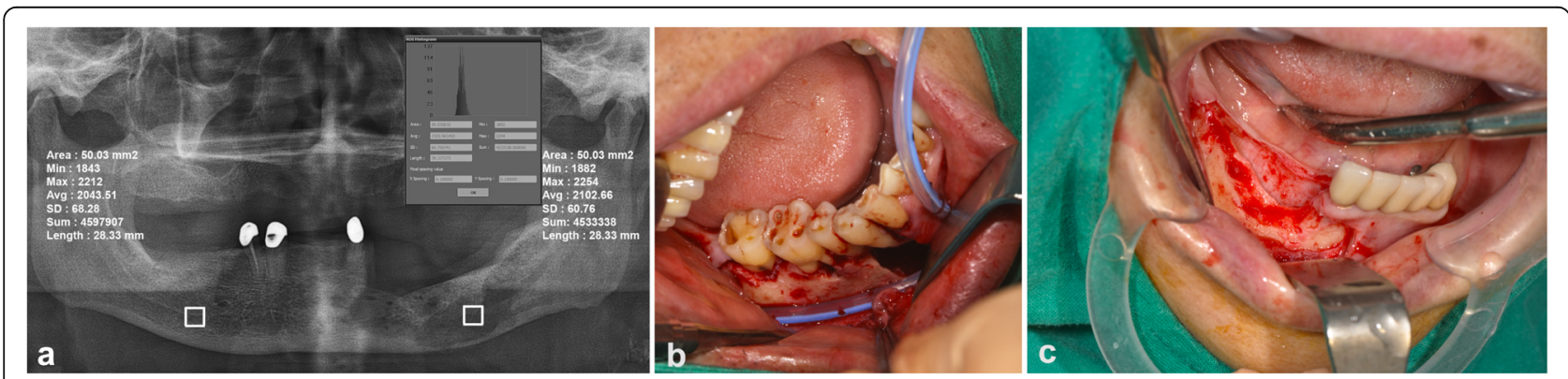

Fig. 1 The radiographic bone density expressed as grayscale values (GSV) were measured by using the easy digitalized panoramic analysis method. Rectangle annotation analysis of INFINITT PACS ${ }^{\circledR}$ (INFINITT Healthcare, Seoul, Korea) was used for measuring the radiographic bone density GSV in suppurative OM as shown in patient No.7. A standard rectangular lesion area of approximately 50 mm² was defined as the region of interest, designated at the center of the OM lesion. The bone density of the contralateral bone in the healthy region was also measured as a reference value to determine the absolute value (a). Intraoperative view of decompressive drain insertion intraorally following surgical management in suppurative type OM with drain (b). Intraoperative view of surgical debridement in sclerosing OM without drain (c) 

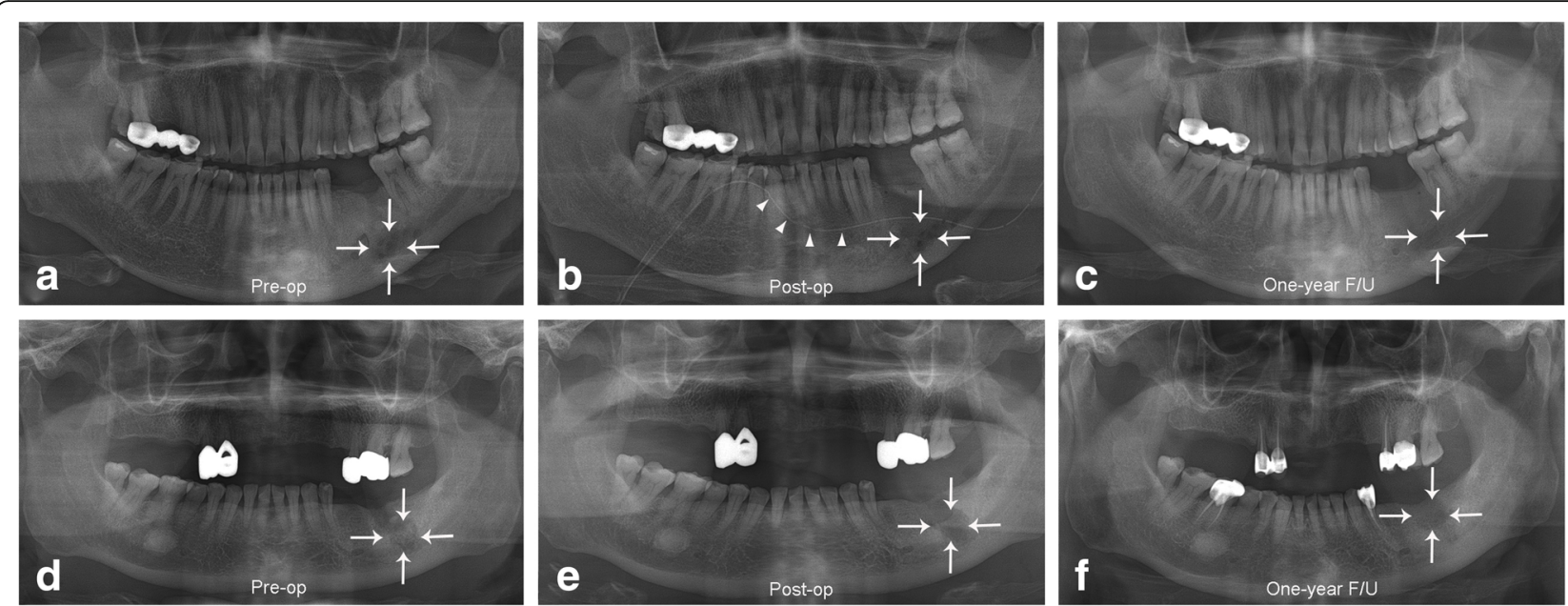

Fig. 2 A panoramic radiograph showing the comparison of sclerosing type OM with and without drainage.. Pre-operative sclerosing OM on panoramic X-ray shows as a dense lesion with increased radiopacity compared to the surrounding healthy bone tissue (marked with arrows) in patient No. 22 (a). Immediate post-operative view following saucerization and drain insertion for decompression (marked with arrowheads). Panoramic X-ray view may show only the lingual wall of mandibular body with minimum soft tissue image. b. Following the decortication procedure, the sclerosing OM could be healed uneventfully with bone turnover, resulting in the decrease of bone density in the sclerotic area (the decrease of GSV) with minimal difference from the surrounding healthy bone tissue at one-year follow-up (c). Pre-operative panoramic radiograph showing sclerotic OM in the left posterior mandible region in patient No.67 (d). Immediate post-operative view without drain (e). One-year follow-up view (f)

commonly affected $(n=19,44.1 \%)$, followed by the left posterior mandible $(n=16,37.2 \%)$, the anterior mandible $(n=3,7 \%)$, the left posterior maxilla $(n=3,7 \%)$, the anterior maxilla $(n=1,2.3 \%)$, and the right posterior maxilla $(n=1,2.3 \%)$. In our study, a total of 29 patients underwent decompression with drainage following the surgical procedures (Fig. 1b), and 14 patients without drainage were included (Fig. 1c). The patients were further categorized based on subtype of disease and use of a drain. In the sclerosing type OM group, 14 (32.6\%) patients received decompression with drainage, while nine patients were treated without drainage (Fig. 2) (20.9\%). In the suppurative type OM group, 15 patients received decompression treatment with drainage (34.9\%), while five patients were treated without drainage (11.6\%) (Fig. 3). The most frequently performed
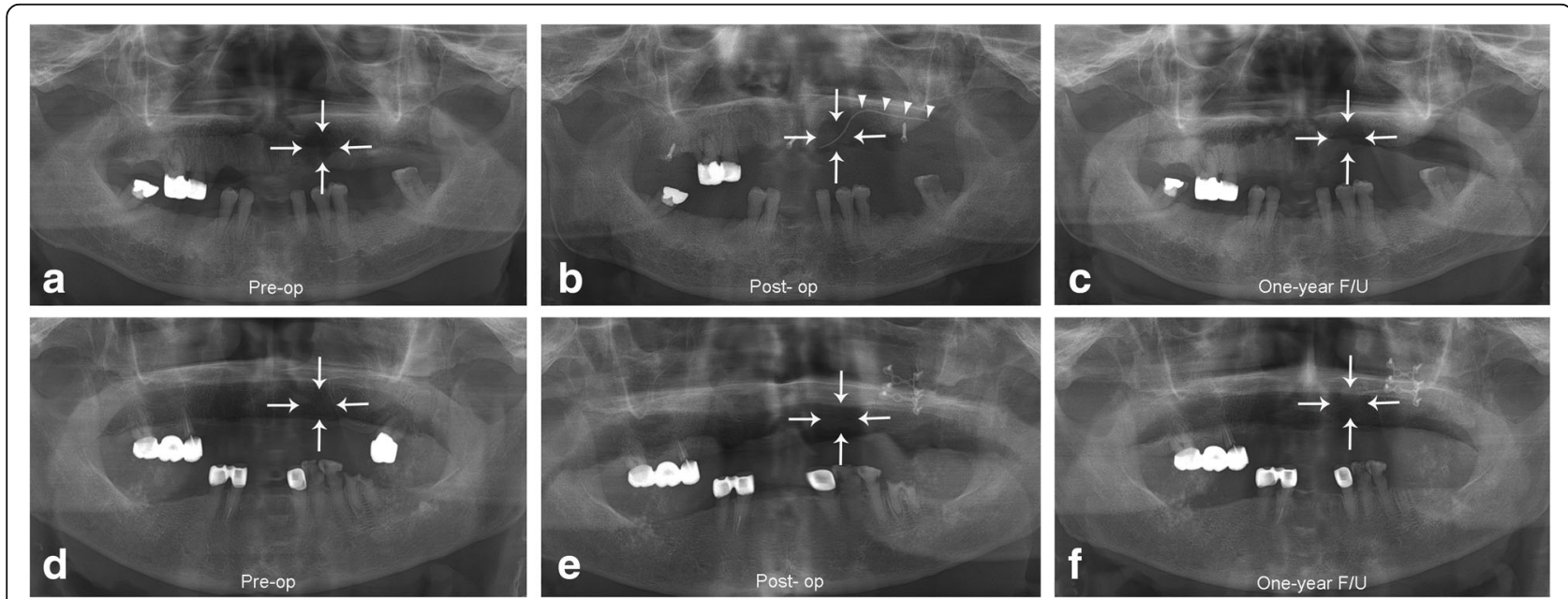

Fig. 3 A panoramic radiograph comparing suppurative type OM with and without drainage. Pre-operative radiograph showing a suppurative OM lesion in the left posterior maxilla region (marked with arrows) in patient No. 30 (a). Immediate post-operative view following saucerization and drain insertion for decompression (marked with arrowheads) (b). A one-year follow-up radiograph showing bony healing (c). Pre-operative panoramic radiograph showing sclerotic OM in the left posterior maxilla region in patient No. 23 (d). Immediate post-operative view without drain (e). One-year follow-up view (f) 
Table 1 Clinicopathologic features of OM in this study

\begin{tabular}{|c|c|c|c|c|}
\hline Variables & $\begin{array}{l}\text { Sclerosing OM } \\
\text { with drain }(n=14)\end{array}$ & $\begin{array}{l}\text { Sclerosing OM } \\
\text { without drain }(n=9)\end{array}$ & $\begin{array}{l}\text { Suppurative OM with } \\
\text { drain }(n=15)\end{array}$ & $\begin{array}{l}\text { Suppurative OM without } \\
\text { drain }(n=5)\end{array}$ \\
\hline \multicolumn{5}{|l|}{ Sex } \\
\hline Male & 4 & 7 & 5 & 1 \\
\hline Female & 10 & 2 & 10 & 4 \\
\hline Age & $67.07 \pm 10.83(82-45)$ & $61.89 \pm 13.74(83-37)$ & $62.80 \pm 17.29(80-12)$ & $66.60 \pm 19.76(87-40)$ \\
\hline \multicolumn{5}{|l|}{ Signs and symptoms } \\
\hline Swelling & $6(42.86 \%)$ & $6(66.67 \%)$ & $11(73.33 \%)$ & $3(60.00 \%)$ \\
\hline Pain & 9 (64.29\%) & $5(55.55 \%)$ & $7(46.67 \%)$ & $2(40.00 \%)$ \\
\hline Fistula & $4(28.57 \%)$ & - & $5(33.33 \%)$ & $1(20.00 \%)$ \\
\hline Pus discharge & $4(28.57 \%)$ & $3(33.33 \%)$ & $5(33.33 \%)$ & $1(20.00 \%)$ \\
\hline Tenderness & $1(7.14 \%)$ & - & $2(13.33 \%)$ & $1(20.00 \%)$ \\
\hline Exposed necrotic bone & - & - & $1(6.67 \%)$ & - \\
\hline Discomfort & $1(7.14 \%)$ & $1(11.11 \%)$ & - & $1(20.00 \%)$ \\
\hline \multicolumn{5}{|l|}{ Etiology } \\
\hline Odontogenic infection & $11(25.58 \%)$ & $3(6.97 \%)$ & $8(18.6 \%)$ & $1(2.32 \%)$ \\
\hline Post-extraction complication & $4(9.30)$ & $5(11.63 \%)$ & $5(11.63 \%)$ & $3(6.97 \%)$ \\
\hline Infected fracture & - & - & $1(2.32 \%)$ & - \\
\hline Sinusitis & $2(4.5 \%)$ & - & - & - \\
\hline Number of interventions & $1.14 \pm 0.36$ times & $1.22 \pm 0.44$ times & $1.06 \pm 0.01$ times & $1.40 \pm 0.54$ times \\
\hline \multicolumn{5}{|l|}{ Systematic disease } \\
\hline Hypertension & $8(57.14 \%)$ & $4(44.44 \%)$ & $8(53.33 \%)$ & $1(20.00 \%)$ \\
\hline Diabetes mellitus & $2(14.29 \%)$ & $1(11.11 \%)$ & $3(20.00 \%)$ & $2(40.00 \%)$ \\
\hline Bleeding tendency & $2(14.29 \%)$ & - & $3(20.00 \%)$ & - \\
\hline Cardiovascular & $2(14.26 \%)$ & - & - & - \\
\hline Osteoporosis & $3(21.43 \%)$ & $2(22.22 \%)$ & $2(13.33 \%)$ & $1(20.00 \%)$ \\
\hline Rheumatoid arthritis & - & - & $1(6.67 \%)$ & - \\
\hline Smoking & $1(7.14 \%)$ & - & $1(6.67 \%)$ & - \\
\hline Time of drain in situ & $2.14 \pm 1.35$ days & - & $3.87 \pm 1.53$ days & \\
\hline
\end{tabular}

surgical treatment was saucerization $(n=34,79 \%)$ followed distantly by sequestrectomy $(n=7,16.2 \%)$ (Table 1).

\section{Cases of radiographic bone density in OM of the jaw}

We compared radiographic bone density changes between the groups immediately post-operatively and at the three-month, six-month, and one-year follow-ups. The immediate post-operative and three-month followup radiographic density results showed no significant differences between sclerosing type with and without drainage or between suppurative type with and without drainage.

In the sclerosing OM with drain, bone density decreased with statistical significance from $249.1 \pm 147.41$ to $122.94 \pm 100.08(p<0.05)$ at the one-year follow-up. For evaluating the internal structure, the term 'predominantly' means more than half the lesion. For instance, where the internal structure of the lesion is predominantly more radiopaque than the surrounding bone, the lesion should be called sclerotic [12]. Therefore, bone healing in sclerotic OM indicates the normalization of the bone architecture, which results in radiopacity decrease of the lesion, making little difference from the surrounding healthy bone. In the sclerosing OM without drain, bone density differences changed from $291.28 \pm$ 117.36 GSV to $272.68 \pm 126 \mathrm{GSV}(p>0.05)$ at the oneyear follow-up. In the OM cases, statistical significance was observed between radiographic bone density results in the sclerosing type with drain and without drain at the six-month follow-up $(p<0.05)$ and one-year followup $(p<0.05)$ (Table 2) (Fig. 4).

In the suppurative type OM with drain group, statistically significant change was observed between the post-operative and one-year follow-up radiographic bone density $(p<0.05)$. Significant difference was 
Table 2 Comparison of radiographic bone densities between sclerosing type with drain and sclerosing type without drain at different time periods

\begin{tabular}{llll}
\hline Sclerosing OM & With drain & Without drain & $P$ - value \\
\hline Immediately post-operative & $249.1 \pm 147.41$ & $291.28 \pm 117.36$ & 0.478 \\
Three-month follow-up & $180.83 \pm 119.99$ & $268.67 \pm 138.33$ & 0.121 \\
Six-month follow-up & $155.13 \pm 109.65$ & $261.4 \pm 128.25$ & $0.046^{*}$ \\
One-year follow-up & $122.94 \pm 100.08$ & $272.68 \pm 126$ & $0.005^{*}$ \\
\hline
\end{tabular}

*Statistical significance was performed with the t-test test $(p<0.05)$. ANOVA test to check if there are statistical significance between the groups at different time points. The absolute values for radiographic bone densities are presented as mean \pm standard deviation grayscale value (GSV)

observed between the suppurative OM with drain and without drain groups at the one-year follow-up, with a mean difference of $-149.73 \pm 47.28$ GSV $(p<0.05)$ (Table 3) (Fig. 5).

\section{Laboratory results in $\mathrm{OM}$ of the jaw}

We analyzed pre- and post-operative blood tests including laboratory markers for systemic inflammation such as WBC, $\mathrm{Hb}$, Hct, and ANC, as well as ESR. While there was no statistical difference in $\mathrm{Hb}$, Hct, ANC or WBC pre- and post-operatively, the ESR exhibited statistically significant results over time in the $\mathrm{OM}$ with drain compared to the OM without drain $(p<0.05)$ (Table 4$)$.

\section{Discussion}

The currently acknowledged treatment for $\mathrm{OM}$ of the jaw is a combination of antibiotic therapy and surgical management with hyperbaric oxygen treatment. Between 500 and 700 species of bacteria have been identified in the human mouth [13]. Therefore, it should be no surprise that $\mathrm{OM}$ of the jaw is a polymicrobial infection. In a normal state, bone is highly resistant to infection. However, various in vitro experiments, animal models, and clinical studies have demonstrated that $S$. aureus is infection of osteoblasts and osteocytes with intracellular persistence within the bone. In this state, antibiotic delivery is limited due to the compromised local vascular system, and pathogenic bacteria may persist after debridement of necrotic bone tissue, which can be explained by the biofilm theory [8].

Recent trends in therapy of OM of the jaw are associated with early diagnosis and aggressive treatment options. This results in osseous defects and need for repair. In this case, more rapid wound healing and bone regeneration are required. The negative pressure decompressive drainage system is a cost-effective, timeefficient, and highly effective treatment option with low associated morbidity in cases of OM of the jaw. It is theorized that more enhanced bone regeneration and eradication of pathogenic bacteria can be achieved. With the use of this system, persisting pathogenic bacteria can be eliminated through negative pressure suction. With promotion of vasculogenesis, the antibiotic effects are enhanced. Passive and active negative pressure drainage systems have been routinely used for post-operative head and neck management. Although many surgeons

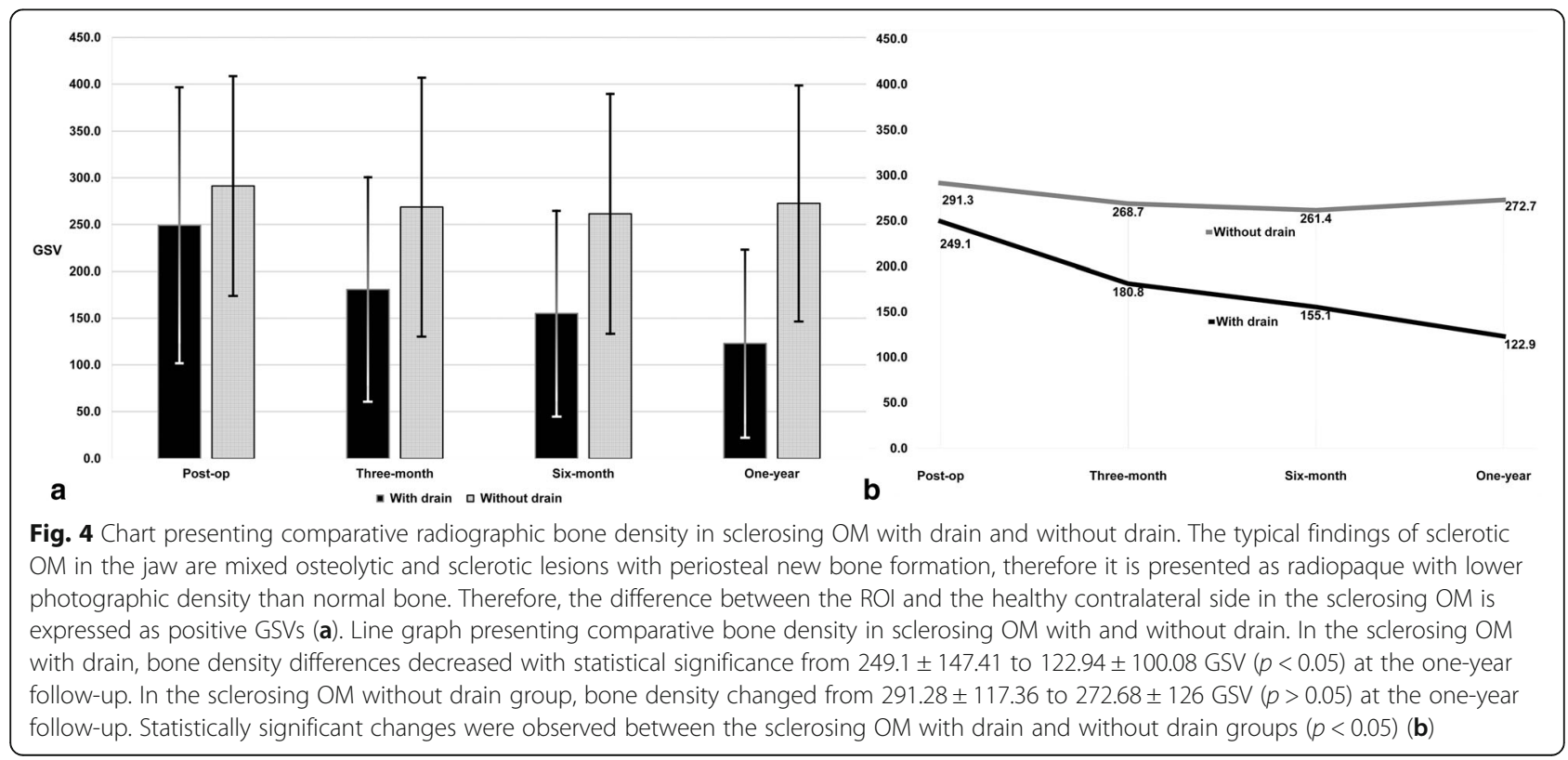


Table 3 Comparison of radiographic bone densities between suppurative type with drain and suppurative type without drain at different time periods

\begin{tabular}{llll}
\hline Suppurative OM & With drain & Without drain & $P$ - value \\
\hline Immediately post-operative & $-182.06 \pm 86.38$ & $-139.2 \pm 78.02$ & 0.339 \\
Three-months follow-up & $-133 \pm 99.89$ & $-149.61 \pm 65.82$ & 0.735 \\
Six-months follow-up & $-99.91 \pm 100.22$ & $-157.5 \pm 103.34$ & 0.285 \\
One-year follow-up & $-34.51 \pm 58.99$ & $-138.21 \pm 93.17$ & $0.010^{*}$ \\
\hline
\end{tabular}

* Statistical significance was performed with the t-test test $(p<0.05)$. ANOVA test to check if there are statistical significance between the groups at different time points. The absolute values for radiographic bone densities are presented as mean \pm standard deviation grayscale value (GSV)

use these drainage systems, there is a lack of clinical data on the bone regenerative aspects of decompression at OM sites.

The osteogenic properties of active negative pressure drainage have been widely reported in clinical cases of various odontogenic cysts, where they are used as a sole treatment in young developing patients where radical treatment should be avoided [3]. Despite the increasing number of reports on successful treatment of odontogenic cysts with decompression, there are no studies on the negative pressure drainage effects on OM of the jaw bone. The aim of our retrospective study was to evaluate the effectiveness of decompression using active negative pressure drainage in the bone healing process following surgical treatments in the OM of the jaw cases.

With regard to the demographic findings, OM lesions were most commonly found in female patients $(n=31$, $72 \%)$, with the posterior mandible being the most prevalent site $(n=35,81.39 \%)$. These results are consistent with previous studies regarding the body or angle of the mandible as the most prevalent site of infection [7]. Accordingly, $\mathrm{OM}$ in the maxilla is less frequent than that of the mandible, mainly due to the extensive maxillary blood supply [16].

In a recent study, quantitative assessment of panoramic radiograph using the PACS program was proven to be useful for early diagnosis and classification of OM in the jaw. According to this method, new patients showing symptoms can be considered suspicious of OM in the jaw with an average difference GSV greater than 54.49 and minimum value less than 31 or a difference average GSV between 12.81 and 54.49 and a difference minimum value of 39 [17]. Therefore, we used the EDPA method to evaluate the progress of bone healing by measuring radiographic bone density expressed as GSV. Panoramic radiographs are a major non-invasive method for detecting bone formation in a healing osseous defect. Bone healing is visualized by an increase in radiopacity, resulting in higher optical density [15]. However, in sclerosing type OM, the bone density is increased in lesions with high radiopacity [12]. In this case, the decrease of radiopacity compared to the initial density measurements is indications of normal bone healing in sclerotic type $\mathrm{OM}$ of the jaw. Significant results of

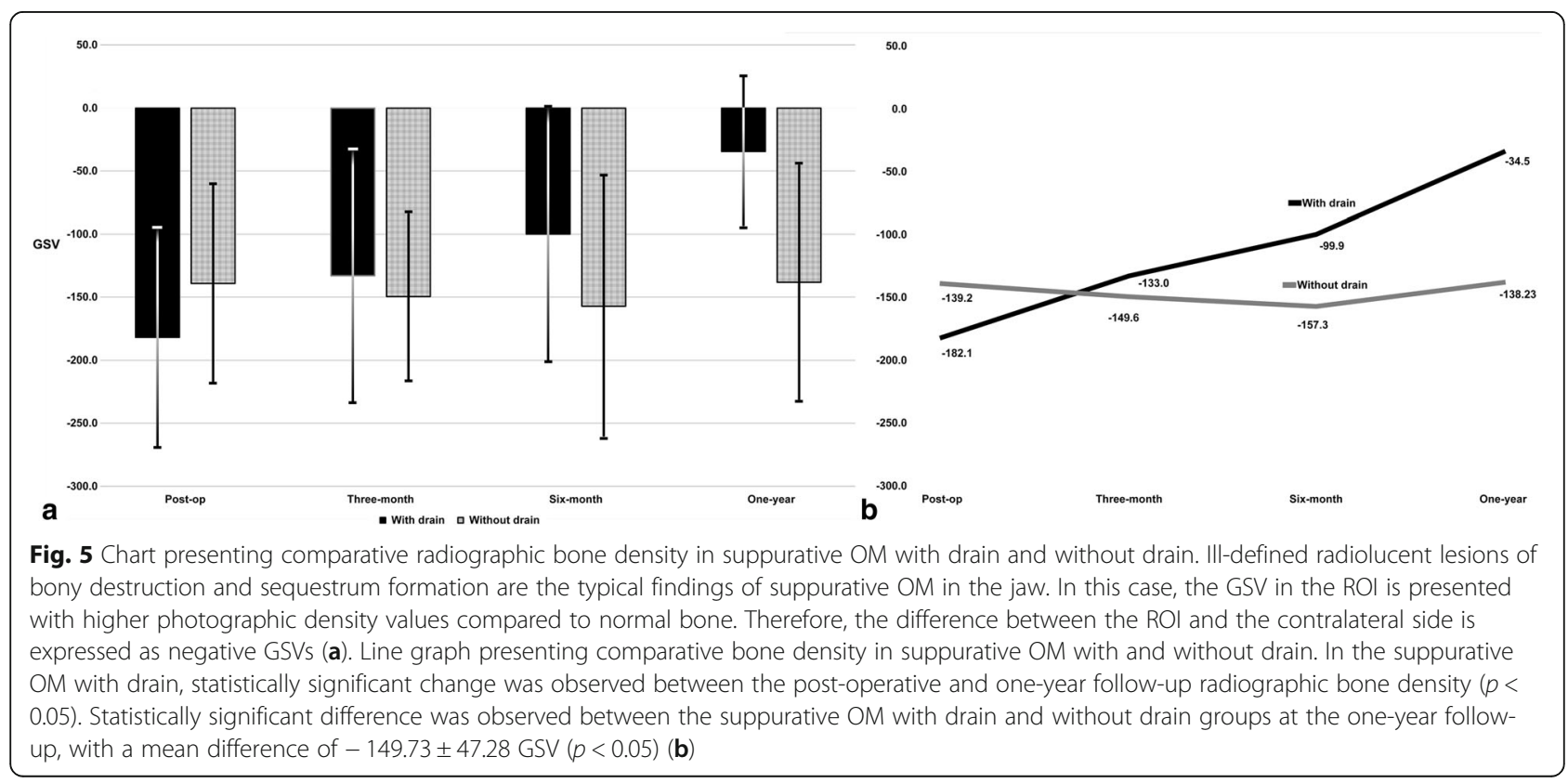




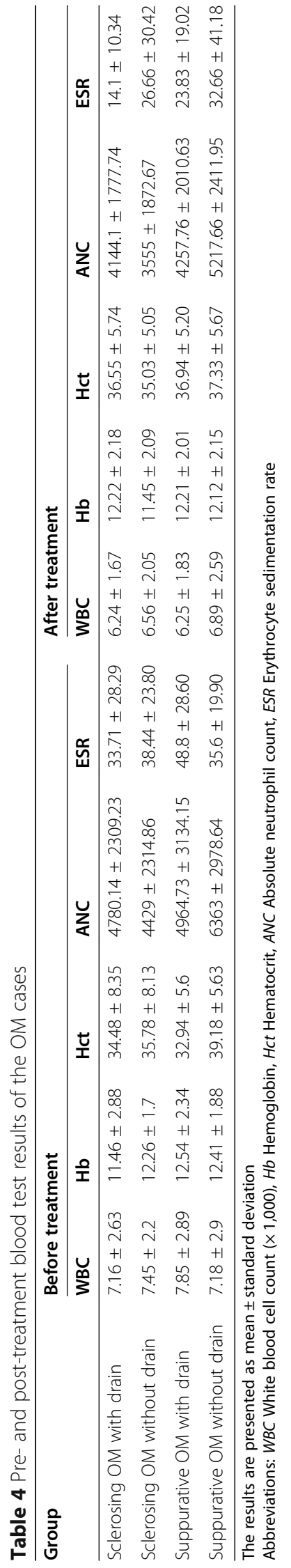


reduction in density differences in the sclerosing $\mathrm{OM}$ cases site were observed at the six-month and one-year follow-ups. The sclerosing type with drain group exhibited a significant decrease in radiographic bone density differences compared to the sclerosing type without drain group at the six-month and one-year follow-ups $(p<0.05)$. Suppurative type OM of the jaw with drain demonstrated significant bone healing results of increased radiopacity at the affected lesion at one-year follow-up.

In this study it was found a significant difference in prognosis after 1-5 days of decompressive drainage at one-year follow-up. Although there was no significant difference in bone healing at three and six-months between the groups, the bone healing process was more gradual and consistent in the decompressive drainage groups. The surgical treatment included debridement of the necrotic and infected bone tissues from the marrow bone sparing the healthy periosteum and cortical bone walls as the sources of osteogenesis for bone regeneration. Research have shown that that suction drainage can expand the capillary diameter of wound, improving the microcirculation of wound, which brings more growth factors to the wounds and promotes the proliferation of granulation tissue [18]. Negative pressure suction drainage can promote the expression of multifarious repair signals and wound healing genes and increase the number of various growth factors and enzymes on the wound surface and surroundings, thereby promoting epithelial regeneration [19]. It should be noted that the complete bone healing of jawbone defects takes considerable amount of time. According to a clinical study by Hren et al., spontaneous bone healing of large bone defects occurred after 1 year, where $46 \%$ of bone gain was achieved after surgical treatment [20]. Thus, more bone healing occurred in cases where decompression drainage was applied compared to that of without drainage.

The sclerosing $\mathrm{OM}$ on panoramic X-ray shows as a dense lesion with increased radiopacity compared to the surrounding healthy bone tissue. The surgical treatment of sclerosing $\mathrm{OM}$ was usually done by decortication and removal of inflammatory debris and followed by drain application and periosteal soft tissue covering. Therefore, panoramic X-ray view may show only the lingual wall of mandibular body with minimum soft tissue image. Following the decortication procedure, the sclerosing OM could be healed uneventfully with bone turnover, resulting in the decrease of bone density in the sclerotic area (the decrease of GSV) with minimal difference from the surrounding healthy bone tissue.

On the other hands, suppurative maxillary OM shows remarkable radiolucency due to soft tissue swelling filled with extensive inflammatory exudate compared to sclerosing
OM. As the suppurative OM became healed after surgical enucleation of inflammatory debris and decompressive drain application, its soft tissue density may be rapidly reduced and affect the GSV of panoramic image by increasing GSV in the negative range compared to those not using drain. However, the present data may indicate surgical treatment of suppurative $\mathrm{OM}$ using decompressive drain can rapidly remove inflammatory exudate from soft tissue and bone marrow compared to that not using decompressive drain during postoperative period.

The use of negative pressure in wound therapy is a wellknown phenomenon that stimulates angiogenesis, improves blood circulation, promotes cell growth of granulation tissue, and accelerates the wound healing process through its mechanical and biological effects in soft tissue. In cases of $\mathrm{OM}$ of the jaw, this method has the major advantage of removing inflammatory exudates, including pathogenic bacteria, from areas of postoperative wound edema. This effectiveness was demonstrated in our previous immunoprecipitation high-performance liquid chromatographic analysis study, where the post-operative exudate obtained from the negative-pressure decompression device in cases of chronic suppurative type OM of the jaw showed an increased inflammatory reaction of innate immunity and slight increase of osteogenesis-related proteins such as osteoprotegerin (OPG) and alkaline phosphatase [21]. Cases of BRONJ demonstrated an elevation of inflammatory signaling and increased expression of angiogenesis-related proteins, i.e., VEGF-A and VEGF-C, and osteogenesis-related proteins, i.e., OPG and osteocalcin [22]. In an ex vivo study by Zhang et al., intermittent negative pressure of $-50 \mathrm{kPa}$ inhibited proliferation of human mesenchymal stem cells (MSCs) and promoted cellular apoptosis. In addition, osteoprotegerin ligand mRNA expression was blocked, and OPG mRNA expression increased, resulting in bone regeneration and inhibition of bone resorption [23]. Bone regeneration was demonstrated in a rabbit skull defect by increasing the expression of VEGF and BMP-2 [24]. Therefore, our current radiologic study is consistent with our previous studies and presents the effectiveness of decompression radiographically.

Before undergoing any surgical procedures, a preoperative laboratory studies such as complete blood count is required for obtaining the necessary information about the patient's general condition and ensuring the safety of the surgical procedures. It is well established that WBC and ESR are accurate indicators for inflammation. Elevated ESR results is especially useful in the diagnosis and follow-up in patients with osteomyelitis and could be used to monitor the response to therapy [25].

No statistical difference in $\mathrm{Hb}$ and Hct was found preand post-operatively, which may indicate the usage of present decompressive drains did not affect de novo 
vascular hemorrhage compared to OM treatment without drain.

In the analysis of inflammatory markers obtained preand post-operatively, suppurative OM showed marked decrease of WBC count by using decompressive drain (79.6\%) compared to not using (97.2\%), while sclerosing OM showed only a little decrease of WBC count by using compressive drain (87.2\%) compared to not using $(88 \%)$. And ANC count was increased in both of sclerosing (86.7\%) and suppurative OMs (85.8\%) treated with decompressive drain compared to those treated without drain, 80.3 and $82 \%$, respectively. These results indicate the possibility of a residual infection in sclerosing OM and a trend of secondary infection through open wound persisted by drain during postoperative period both in sclerosing and suppurative OMs.

The pre- and post-operative ESR results in suppurative type $\mathrm{OM}$ with drain showed significant reduction (48.83\%) compared to that of without drain (91.74\%). Similarly, the ESR results were significantly different in the sclerosing $\mathrm{OM}$ with drain pre- and post-operatively (41.82\%), while the sclerosing OM without drain showed no significant difference (69.3\%). The reduction in increased laboratory marker for inflammation such as ESR to the normal range could be considered an indicator of successful treatment.

The limitation of our study is associated with the EDPA method. The weak point of EDPA method include the use of panoramic radiographs, where many artifacts and errors could be encountered while converting $3 \mathrm{D}$ bone tissue into a $2 \mathrm{D}$ depending on the position, the angle and the timing of the photography. The computed tomography (CT), on the other hand is expresses as Hounsfield Unit (HU) and the radiographic bone density can be readjusted as HU's through a standardization process. Therefore, the results can be used as an absolute value without errors. More precise analysis using periodic CT scan for radiographic bone density measurement and a periodic laboratory data is recommended.

In conclusion, the results of our study indicate positive outcomes of routine use of decompression for management of OM of the jaw. The OM groups with drain exhibited more enhanced bone density compared to the groups without drainage at the six-month and one-year follow-ups. The drain insertion for decompression is effective for the management of sclerosing and suppurative types $\mathrm{OM}$ in the jaw. Decompression using the simple draining tubes such as the silastic drain, penrose drain, Jack-Pratt drain and Hemovac have proven to be easy to use, convenient and economical. The intraorally anchored drain compared to the extraoral drain, has more benefits including no scar formation and more comfort to the patient. Based on our study, it is recommended for oral and maxillofacial surgeons to implement decompression with drain for the management of $\mathrm{OM}$ of the jaw.

\section{Acknowledgements}

Not applicable in this section.

\section{Authors' contributions}

All authors read and approved the final manuscript. BS collected, analyzed, and interpreted the data; and wrote the manuscript. MYE and MHS

participated in the design of this study and coordination of the study. $\mathrm{JHL}$ and SMK provided critical review and substantially revised the manuscript.

\section{Funding}

This study was supported by grant no 03-2019-0043 from the SNUDH Research Fund and by Basic Science Research Program of NRF funded by the Ministry of Education (2017R1D1A1B04029339).

\section{Availability of data and materials}

The datasets used and/or analyzed during the current study are available from the corresponding author on reasonable request.

\section{Declarations}

\section{Ethics approval and consent to participate}

The study procedures received the ethics approval from the School of Dentistry, Seoul National University Institutional Review Board (S-D20160039), and with the 1964 Helsinki declaration and its later amendments or comparable ethical standards. A written informed consent was obtained from all of the patient.

\section{Consent for publication}

Not applicable.

\section{Competing interests}

Buyanbileg Sodnom-Ish, Mi Young Eo, Mi Hyun Seo, Jong Ho Lee, and Soung Min Kim declare that they have no competing interests.

Received: 16 December 2020 Accepted: 6 May 2021

Published online: 22 May 2021

\section{References}

1. Moreno-Rodríguez P, Guerrero LM, Gómez-Delgado A, Castro-Núñez J. Active decompression and distraction sugosteogenesis for the treatment of calcifying odontogenic cyst. Oral Maxillofac Surg. 2020;25:89.

2. Castro-Núñez J. An innovative decompression device to treat odontogenic cysts. J Craniofacial Surg. 2016;27:5.

3. Pogrel MA. Decompression and marsupialization as a treatment for the odontogenic keratocyst. Oral Maxillofac Surg Clin North Am. 2003;15(3): 415-27.

4. Partsch C. Uber kiefercysten. Dtsch Mschr Zahnheilkd. 1892;10:271.

5. Neuschmidt U. Drainierungs-Methode der Kieferzysten. Dtsch Zahnärztl Wschr. 1942;21:287.

6. Castro-Núñez J. Distraction Sugosteogenesis: its biologic bases and therapeutic principles. J Craniofacial Surg. 2018;29:1.

7. Haeffs TH, Scott CA, Campbell TH, Chen Y, August M. Acute and chronic Suppurative osteomyelitis of the jaws: a 10-year review and assessment of treatment outcome. J Oral Maxillofac Surg. 2018;76(12):2551-8.

8. Dym H, Zeidan J. Microbiology of acute and chronic osteomyelitis and antibiotic treatment. Dent Clin N Am. 2017;61(2):271-82.

9. Berglund C, Ekströmer $K$, Abtahi J. Primary chronic osteomyelitis of the jaws in children: an update on pathophysiology, radiological findings, treatment strategies, and prospective analysis of two cases. Case Rep Dent. 2015;2015:152717.

10. Baltensperger MM: A retrospective analysis of 290 osteomyelitis cases treated in the past 30 years at the Department of Cranio-Maxillofacial Surgery Zurich with special recognition of the classification. Verlag nicht ermittelbar; 2003.

11. Baur DA, Altay MA, Flores-Hidalgo A, Ort Y, Quereshy FA. Chronic osteomyelitis of the mandible: diagnosis and management--an institution's experience over 7 years. J Oral Maxillofac Surg. 2015;73(4):655-65. 
12. Orpe EC, Lee L, Pharoah MJ. A radiological analysis of chronic sclerosing osteomyelitis of the mandible. Dentomaxillofac Radiol. 1996;25(3):125-9.

13. Marschall JS, Flint RL, Kushner GM, Alpert B. Management of Mandibular Osteomyelitis with Segmental Resection, nerve preservation, and immediate reconstruction. J Oral Maxillofac Surg. 2019;77(7):1490-504.

14. Caesar BC, Morgan-Jones RL, Warren RE, Wade RH, Roberts PJ, Richardson JB. Closed double-lumen suction irrigation in the management of chronic diaphyseal osteomyelitis: long-term follow-up. J Bone Joint Surg (Br). 2009; 91(9):1243-8.

15. Zhao Y, Liu B, Wang SP, Wang YN. Computed densitometry of panoramic radiographs in evaluation of bone healing after enucleation of mandibular odontogenic keratocysts. Chin J Dent Res. 2010;13(2):123-6.

16. Baltensperger M, Eyrich G. Osteomyelitis of the Jaws: Definition and Classification. In: Baltensperger MM, GKH E, editors. Osteomyelitis of the Jaws. Berlin: Springer Berlin Heidelberg; 2009. p. 5-56.

17. Park MS, Eo MY, Myoung H, Kim SM, Lee JH. Early diagnosis of jaw osteomyelitis by easy digitalized panoramic analysis. Maxillofacial Plastic Reconstructive Surg. 2019;41(1):6

18. Chen SZ, Li J, Li XY, Xu LS. Effects of vacuum-assisted closure on wound microcirculation: an experimental study. Asian J Surg. 2005;28(3):211-7.

19. Banasiewicz T, Borejsza-Wysocki M, Meissner W, Malinger S, Szmeja J, Kościński T, et al. Vacuum-assisted closure therapy in patients with large postoperative wounds complicated by multiple fistulas. Wideochir Inne Tech Maloinwazyjne. 2011;6(3):155-63.

20. Ihan Hren N, Miljavec M. Spontaneous bone healing of the large bone defects in the mandible. Int J Oral Maxillofac Surg. 2008:37(12):1111-6.

21. Kim SM, Eo MY, Cho YJ, Kim YS, Lee SK. Immunoprecipitation high performance liquid chromatographic analysis of healing process in chronic suppurative osteomyelitis of the jaw. J Cranio-Maxillofac Surg. 2018;46(1): 119-27.

22. Kim SM, Eo MY, Cho YJ, Kim YS, Lee SK. Wound healing protein profiles in the postoperative exudate of bisphosphonate-related osteonecrosis of mandible. Eur Arch Otorhinolaryngol. 2017;274(9):3485-95.

23. Zhang Y-G, Yang Z, Zhang H, Wang C, Liu M, Guo X, et al. Effect of negative pressure on human bone marrow mesenchymal stem cells in vitro. Connect Tissue Res. 2010;51(1):14-21.

24. Y-g Z, Yang Z, Zhang H, Liu M, Qiu Y, Guo X. Negative pressure technology enhances bone regeneration in rabbit skull defects. BMC Musculoskelet Disord. 2013;14(1):76.

25. Lavery LA, Ahn J, Ryan EC, Bhavan K, Oz OK, La Fontaine J, et al. What are the optimal cutoff values for ESR and CRP to diagnose osteomyelitis in patients with diabetes-related foot infections? Clin Orthop Relat Res. 2019; 477(7):1594-602

\section{Publisher's Note}

Springer Nature remains neutral with regard to jurisdictional claims in published maps and institutional affiliations.

Ready to submit your research? Choose BMC and benefit from:

- fast, convenient online submission

- thorough peer review by experienced researchers in your field

- rapid publication on acceptance

- support for research data, including large and complex data types

- gold Open Access which fosters wider collaboration and increased citations

- maximum visibility for your research: over $100 \mathrm{M}$ website views per year

At $\mathrm{BMC}$, research is always in progress.

Learn more biomedcentral.com/submissions 